\title{
Sub-Band Processing of Synthetic Aperture Sonar Data
}

\author{
Sérgio Rui Silva ${ }^{1}$, Sérgio Cunha ${ }^{1,2}$, Aníbal Matos ${ }^{1}$, Nuno Cruz ${ }^{1}$ \\ ${ }^{1}$ Porto University, Faculty of Engineering (www.fe.up.pt), ${ }^{2}$ CIMAR (www.cimar.org) \\ Email: \{srui, sergio, anibal, nacruz\}@fe.up.pt
}

\begin{abstract}
High frequency synthetic aperture sonar systems require demanding tolerances in motion errors and medium phase stability. This article proposes a new method that mitigates the problems associated with small wavelength related errors. By dividing the received signal bandwidths in to several smaller ones and conjugate complex multiplying them, a new signal is obtained with longer effective wavelength, thus reducing the impact of motion errors and medium phase fluctuations.
\end{abstract}

Keywords- Synthetic aperture, sonar, signal processing.

\section{INTRODUCTION}

Today's sonar systems strive for higher and higher resolution. This utterly leads to the transmission of higher frequency signals. Besides the obvious impairments on using high frequency signals such as signal attenuation, unknown platform motion errors and stability of phase propagation through the medium become increasingly important. As an example, the synthetic sonar system developed at University of Porto ([7]) uses a transmission frequency of $200 \mathrm{kHz}$ (wavelength of $7.5 \mathrm{~mm}$ ) and a useful bandwidth of $30 \mathrm{kHz}$. This requires having its unknown motion errors below at least $1 \mathrm{~mm}$ to enable accurate synthetic aperture image formation $([1,2])$, after auto-focus. It requires motion errors bounds prior to autofocus to be in the order of magnitude of the wavelength, which is by itself quite demanding.

This article proposes a new technique that mitigates the problems associated with the small wavelength of high frequency sonar signals. By dividing the received signal bandwidth in several smaller bands and conjugate complex multiplying the pulse compressed signals obtained in each band one by the other, a new resulting signal is obtained with an effective longer wavelength corresponding to the frequency difference between the two sub-bands. This longer wavelength effectively reduces the impact of phase fluctuation from the medium and platform motion uncertainties.

Processing the signal trough this technique can be used to obtain directly a coarse final synthetic aperture sonar image, or used in an global contrast optimization auto-focus algorithm $([3,4,7])$ with several steps with increasingly smaller wavelengths. This enables faster convergence through the resulting smother cost function surfaces (less impact of local minima). If a direct image in intended, a resulting loss of range resolution is unavoidable (due to the use of smaller effective bandwidths) and the along-track resolution even further affected (due to the use of a significantly larger effective wavelength while maintaining the path length covered by the sensor while scanning each target point).

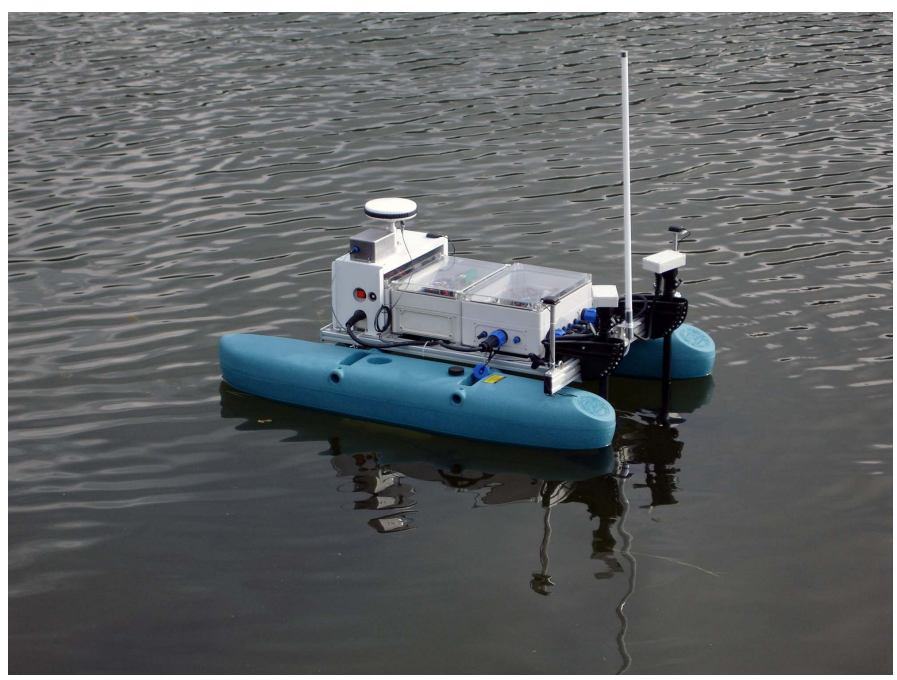

Figure 1: Autonomous boat in operation in operation in the Douro river, Portugal.

This is enough, however, to auto-focus the synthetic aperture image data without the need for any special image features for a system with only one transducer and compensate for rough navigation data, prior to process the image at the native wavelength.

In applications where rougher images are acceptable, this technique can be preferable to using lower frequency transducers directly. Although lower frequencies transducers can produce better along-track resolutions if larger apertures are employed (and this can be difficult in the terrain), their cross-track resolution is easily worse: the bandwidth of lower frequency transducers is significantly worse than that of higher frequencies, even after dividing the bandwidth into sub-bands.

A common RTK differential GPS system has an error in the centimeter level. By using two $15 \mathrm{kHz}$ sub-bands within the $30 \mathrm{kHz}$ bandwidth of the transmitted signals (with a center frequency of $200 \mathrm{kHz}$ ), an equivalent wave of $15 \mathrm{kHz}$. This corresponds to a wavelength of $10 \mathrm{~cm}$, which is larger than the navigation error. For this wavelength and navigation accuracy, correct image formation with auto-focus algorithms are very efficient (only minor phase adjustments are needed for motion compensation). Using an auto-focus algorithm directly with the 
original data (wavelength of $7.5 \mathrm{~mm}$ ) would be extremely complex since adjustments greater than one wavelength would be necessary, causing convergence difficulties due to the numerous local minima. Auto-focus with the original wavelength after motion compensation based on auto-focus using the long wavelength is a much easier task.

The impact of this technique in interferometric height mapping is also analyzed, since using a long wavelength is beneficial in the phase unwrapping stage of the height estimation. A longer wavelength has less wraps for the same height variation and so correct unwrapping of the phase is easily done in the presence of noise.

This technique is illustrated with results obtained with the synthetic aperture sonar system developed at the University of Porto that is using an autonomous boat as its moving platform and is taking effective advantage of high precision satellite GPS navigation system $([5,6])$ and an inertial navigation unit.

The proposed approach is therefore a method to take advantage of both using a short and a long wavelength synthetic aperture sonar system, while using the hardware corresponding to the short wavelength only. The long wavelength processing as a first step allows computing motion compensation information that is relevant for the short wavelength processing step.

\section{Synthetic APERTURe DePENDENCY With WAVELENGTH}

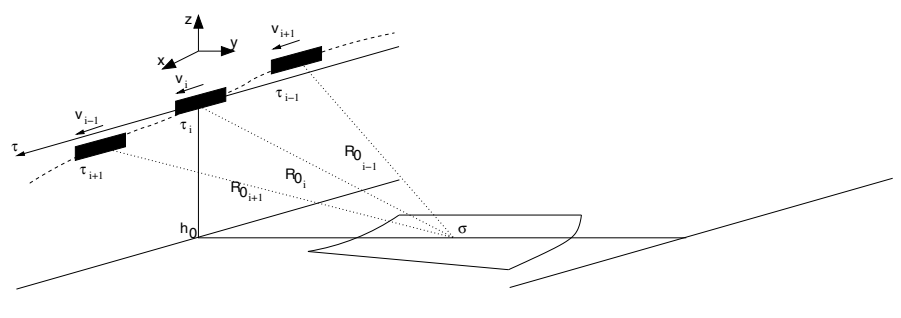

Figure 2: Synthetic Aperture Sonar model.

Remembering the synthetic aperture sonar geometry (Figure 2 ), it is necessary to coherently combine the along-track samples to form an image. Let $\mathrm{ss}_{\mathrm{b}}$ be the baseband transmitted signal, the sequence of echoes given by a point-target will be:

$$
e e_{b}(t, \tau)=s s_{b}\left(t-t_{v}(\tau)\right) e^{-j 2 \pi f_{0} t_{v}}
$$

Where $t_{v}(\tau)$ is the time-of-flight and is given by:

$$
t_{v}(\tau)=\frac{2}{c} \sqrt{\left(x_{p}-x_{0}\right)^{2}+\left(y_{p}-y_{0}\right)^{2}+\left(z_{p}-z_{0}\right)^{2}}
$$

The point-target has coordinates $\left(\mathrm{x}_{0}, \mathrm{y}_{0}, \mathrm{z}_{0}\right)$ and $\left(\mathrm{x}_{\mathrm{p}}, \mathrm{y}_{\mathrm{p}}, \mathrm{z}_{\mathrm{p}}\right)$ are the coordinates of the sonar platform position at each along-track sampling instant $\tau$. In the absence of motion errors, $\mathrm{y}_{\mathrm{p}}$ and $\mathrm{z}_{\mathrm{p}}$ will be zero and $\mathrm{x}_{\mathrm{p}}=\mathrm{v} \tau$, where $v$ is the sonar medium velocity.
The target will be seen by the sonar during the time it is inside the aperture ( $3 \mathrm{~dB}$ lobe) of the transducer, which is approximately given by:

$$
\theta_{3 d B} \approx \frac{\lambda}{D}
$$

The array spacing from Nyquist spatial sampling and classical array theory is $\lambda / 2$ (two way equivalent $2 \pi$ phase shift), which means that for angles of arrival of a wave-front the inter-element phase difference must be less than $2 \pi$ ([8]).

This is also true for motion errors. To correctly form a synthetic aperture the platform position must be known within $1 / 8$ of a wavelength so the echoes can be coherently combined with negligible image deterioration $([9,10,12])$.

Another perspective would be to consider the array spacing to be given by a Pulse repetition Frequency (PRF) that is at least equal the maximum Doppler shift experienced by a target. The Doppler shift $f_{D}$ is related to the radial velocity $\mathrm{v}_{\mathrm{r}}$ by:

$$
f_{D}=\frac{2 v_{r}}{\lambda}=\frac{2 v \sin \theta(\tau)}{\lambda}
$$

The maximum radial velocity is obtained at the beam edge and so the lower bound for the PRF is:

$$
P R F \geq \frac{2 v \sin \theta_{3 d B}}{\lambda} \approx \frac{2 v \lambda / D}{\lambda}=2 \frac{v}{D}
$$

The along-track resolution is independent of the range and wavelength. This results from the fact that for a transducer with a fixed length $\mathrm{D}$, the synthetic aperture length $\mathrm{D}_{\mathrm{SA}}$ will be given, approximately, by:

$$
D_{S A} \approx 2 R_{0} \theta_{3 d B}=2 R_{0} \frac{\lambda}{D}
$$

Where $\mathrm{R}_{0}$ is the distance to the center of the scene.

This than gives the classical synthetic aperture along-track resolution $\delta_{\mathrm{AT}}$ formula:

$$
\delta_{A T} \approx R_{0} \theta_{S A}=R_{0} \frac{\lambda}{D_{S A}}=R_{0} \frac{\lambda}{2 R_{0} \frac{\lambda}{D}}=\frac{D}{2}
$$

We see here that the phase relations that enable the synthetic array formation are tightly related to the wavelength of the signal and the effective synthetic array length. Normally these two values are interconnected due to the transducers real aperture width, but can be explored to mitigate some of the problems inherent to synthetic aperture.

\section{SUB-BAND PROCESSING}

Motion errors are critical to synthetic aperture image formation. Path deviations can be effectively corrected through the use of a back-projection algorithm if each along-track sampling position is known through the use of precise navigation systems such as a RTK-DGPS/INS or a DPCA/INS. Nevertheless, the use of high-frequency signals imposes very 
tight restrictions to the uncertainty of the along-track positions given by these systems.

The goal of sub-band processing is to reduce the effects phase errors due to motion or other sources in very susceptible signals such as the ones with very short wavelength.

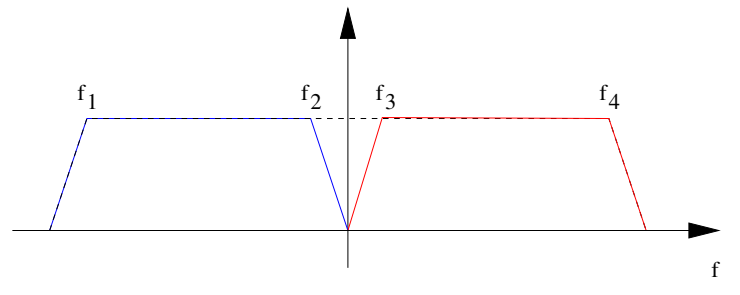

Figure 3: Chirp signal division schematic.

Considering a chirp signal with a spectrum as depicted in Figure 3:

$$
p_{m}=\operatorname{rect}\left(\frac{t}{T}\right) \cdot e^{2 \pi\left(\frac{f_{4}-f_{1}}{T}\right) t^{2}}
$$

The echo data can be modeled by a summation of delay versions of this signal.

It is possible to divide the signal in several parts and thus its bandwidth into several sub-bands. Without loss of generality the case of the division by 2 is considered here:

$$
\left\{\begin{array}{l}
p_{m_{1}}=\operatorname{rect}\left(\frac{t+T / 4}{T / 2}\right) \cdot e^{2 \pi\left(\frac{f_{2}-f_{1}}{T / 2}\right) t^{2}} \\
p_{m_{2}}=\operatorname{rect}\left(\frac{t-T / 4}{T / 2}\right) \cdot e^{2 \pi\left(\frac{f_{4}-f_{3}}{T / 2}\right) t^{2}}
\end{array}\right.
$$

Each of these signals will now have its own center frequency. Multiplying one chirp by the conjugate of the other one gets:

$$
p_{m_{e}}=\operatorname{rect}\left(\frac{t}{T / 2}\right) \cdot e^{2 \pi\left(\frac{\left(f_{4}-f_{2}\right)-\left(f_{3}-f_{1}\right)}{T / 2}\right) t^{2}}
$$

After frequency recentering and pulse compression with the corresponding sub-chirp, a point target will have an echo response given by:

$$
\left\{\begin{array}{l}
S S_{b_{1}}=\left\|S s_{b_{1}}\right\| e^{-2 \pi\left\langle f_{2}, f_{1}\right\rangle t_{v}(\tau)} \\
S s_{b_{2}}=\left\|S s_{b_{2}}\right\| e^{-2 \pi\left\langle f_{4}, f_{3}\right\rangle t_{v}(\tau)}
\end{array}\right.
$$

This new signal will necessarily have lower cross-track resolution which is given by its lower bandwidth:

$$
\delta_{X T}=\frac{c}{2 B W}
$$

By conjugate multiplying one by the other, one obtains a signal such that its wavelength corresponds to the beat frequency of the central frequencies of the two sub-bands:

$$
\begin{aligned}
& S S_{b_{e}}=S S_{b_{2}} \cdot S S_{b_{1}}{ }^{*}= \\
& =\left\|S S_{b_{2}}\right\| \cdot\left\|S S_{b_{1}}\right\| \cdot e^{-2 \pi\left(\left\langle f_{2}, f_{1}\right\rangle-\left\langle f_{4}, f_{3}\right\rangle\right) t_{v}(\tau)}= \\
& =\left\|S S_{b_{e}}\right\| \cdot e^{-2 \pi f_{e} t_{v}(\tau)}
\end{aligned}
$$

This new equivalent (and longer) wavelength enables higher robustness to phase errors.
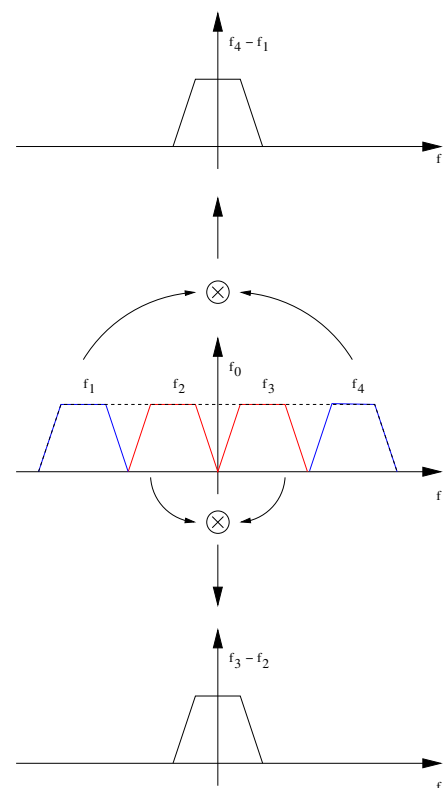

Figure 4: Grouping of the several sub-bands to form a new signals with longer wavelength.

As can be seen in the schematic (Figure 4), the original echo data is divided into several bands (four in this example). Each sub-band originates a new set of echo data which is frequency recentered and pulse compressed with the corresponding chirp signal. Combining the each set of echoes one obtains two equivalent signals with longer wavelengths than the original one $\left(f_{4}-f_{1}\right.$ and $\left.f_{3}-f_{1}\right)$. With different divisions of the original bandwidth it is possible to obtain different wavelengths.

Besides the obvious cross-track resolution loss, the alongtrack resolution also decreases. This is because the longer wavelength is not compensated by a longer along-track integration path width is maintained. As can be seen in Figure 5 , the original synthetic aperture length is not changed (the target is still visible through $\left[\tau_{1}, \tau_{2}\right]$ ) and the new Doppler frequency is:

$$
f_{D_{e}}=\frac{2 v_{r}}{\lambda_{e}}=\frac{2 v \theta(\tau)}{\lambda_{e}}=, \tau \in\left[\tau_{1}, \tau_{2}\right] \Rightarrow \frac{2 v \theta_{3 d B}}{\lambda_{e}} \leq \frac{2 v \theta_{3 d B}}{\lambda}
$$

This equivalent center frequency is lower, which gives rise to a lower Doppler shift and thus a lower resolution. 


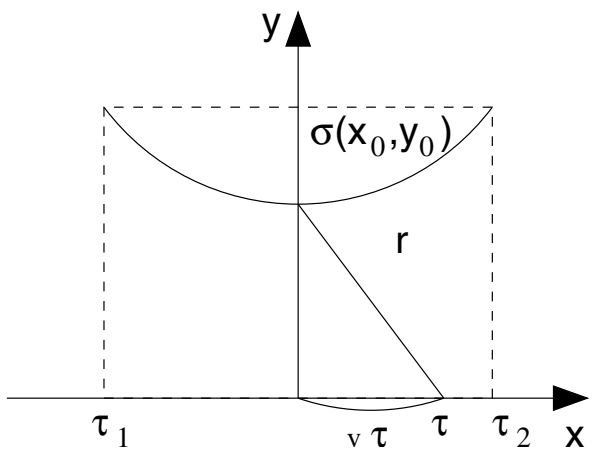

Figure 5: Synthetic aperture length.

The along-track resolution is inferior by a factor equal to ratio between the original wavelength and the actual effective wavelength:

$$
\delta_{A T_{e}}=R_{0} \frac{\lambda_{e}}{D_{S A}}
$$
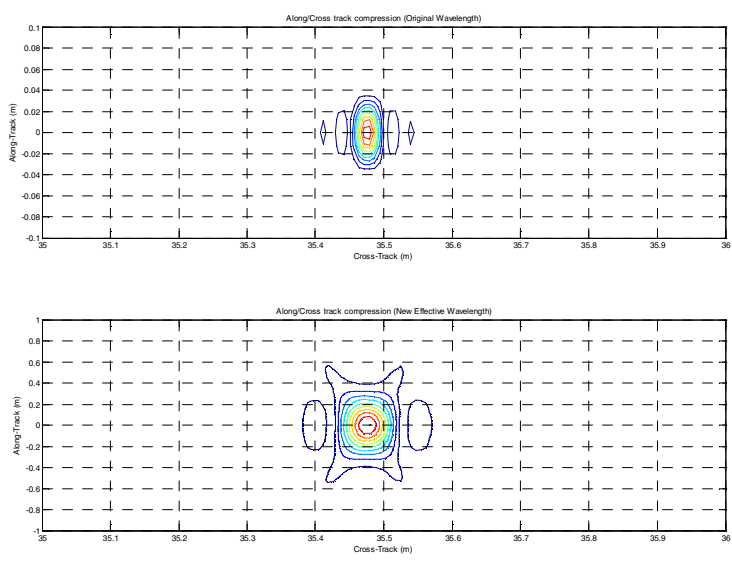

Figure 6: Resolution decrease in along/cross track when using sub-band processing.

Figure 6 shows the result of a simulation where a single target was processed using the original data and the data after sub-band division in two bands. The original data center frequency and bandwidth is respectively $200 \mathrm{kHz}$ and $30 \mathrm{kHz}$. The cross-track resolution is lower by a factor of 2 and the along-track resolution lower by a factor of, approximately, 13 .
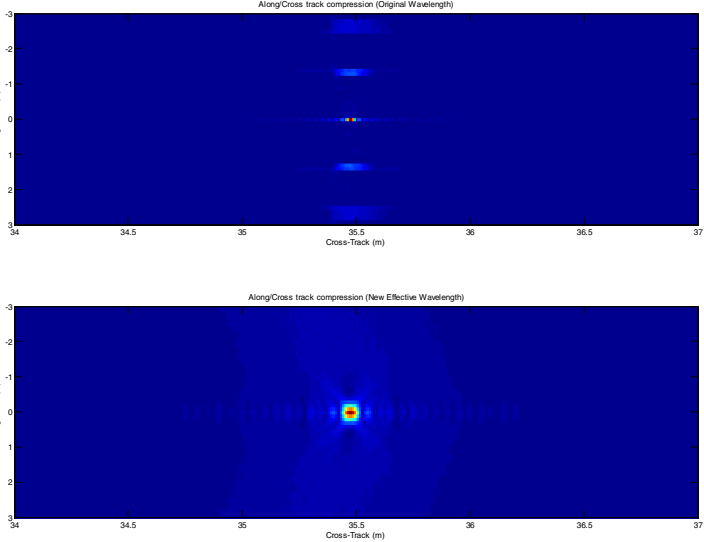

Figure 7: Single target under-sampled in the along-track direction.

Since the wavelength is longer, the aliasing effects that occur when the swath is under-sampled in the along-track direction are attenuated or even disappear if the new wavelength meats the new sampling criteria. This can be seen in the simulation results present in Figure 7. This is again a result of the fact that the integration time is not modified but the Doppler frequency is decreased.

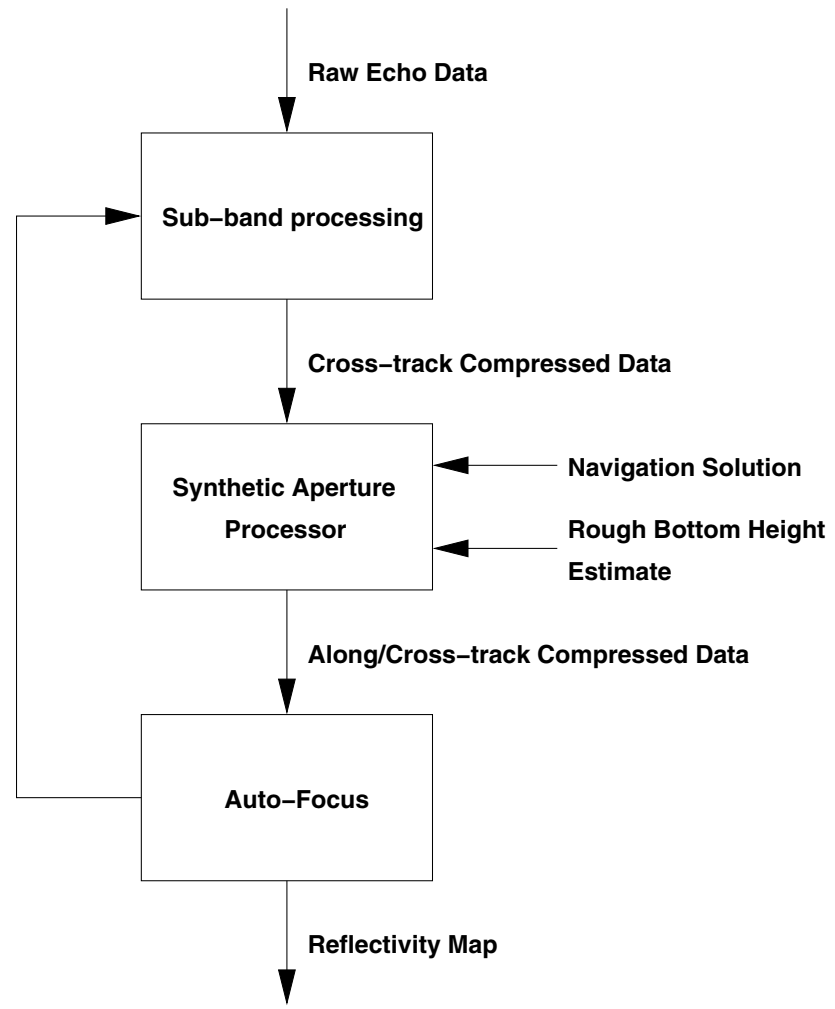

Figure 8: Signal flow diagram for the use of the sub-band algorithm within a synthetic aperture processor. 
As sub-band processing is a step that leads to the pulsecompressed images, it is possible to use this method with any synthetic aperture processor that can use pulse compressed data This includes the family of back-projection algorithms, the wave-number algorithm and the inverse scaled Fourier transform algorithm.

Figure 8 shows a signal flow diagram that situates the subband algorithm within a synthetic aperture processor.

\section{Auto-Focus}

The use of global auto-focus algorithm presents several advantages for synthetic aperture sonar image enhancing. Common auto-focusing algorithms require restrict along-track sample rates equal or higher than the Nyquist sample rate. This imposes unpractical velocity constrains, especially for system that use few receivers (as is the case with the sonar system described here). It is not possible to obtain micro-navigation from an under-sampled swath or to perform displaced center phase navigation with only one transducer. So, with these impairments, global auto-focus algorithms are required in this type of sonar systems. Moreover, global auto-focus algorithms correct not only phase errors due to navigation uncertainties, but also phase errors that are due to medium fluctuations.

The back-projection algorithm uses the available navigation solution to place the echo data in the correct image coordinates Assuming that navigation errors are small compared to the range to the center of the image, further adjustments to the echo data placement can be made using only a small adjustment to the mean range (Figure 9). So, each along-track sampling position has it space coordinates and a variable, $\Delta \mathrm{r}$, that affects all the data that is back-projected. This adjustment is responsible for the integration in the along-track direction.

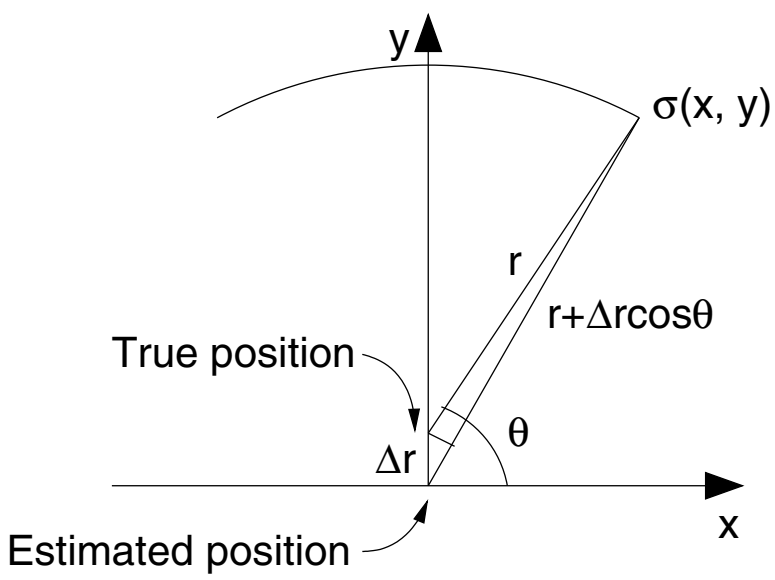

Figure 9: Position adjustment approximation for auto-focus.

Nevertheless, these errors are seldom smaller than the original signal wavelength, and so create a solution surface that is difficult to search for the optimum set of parameters.
Using sub-band processing, it is possible to divide the signal bandwidth into several sub-bands and combine them in to signals with different wavelengths. At the first step, a large wavelength is used since the expected motion correction is also large. After achieving a predefined level of image quality, the auto-focus algorithm then proceeds by using a smaller wavelength and the previous estimated position parameters.

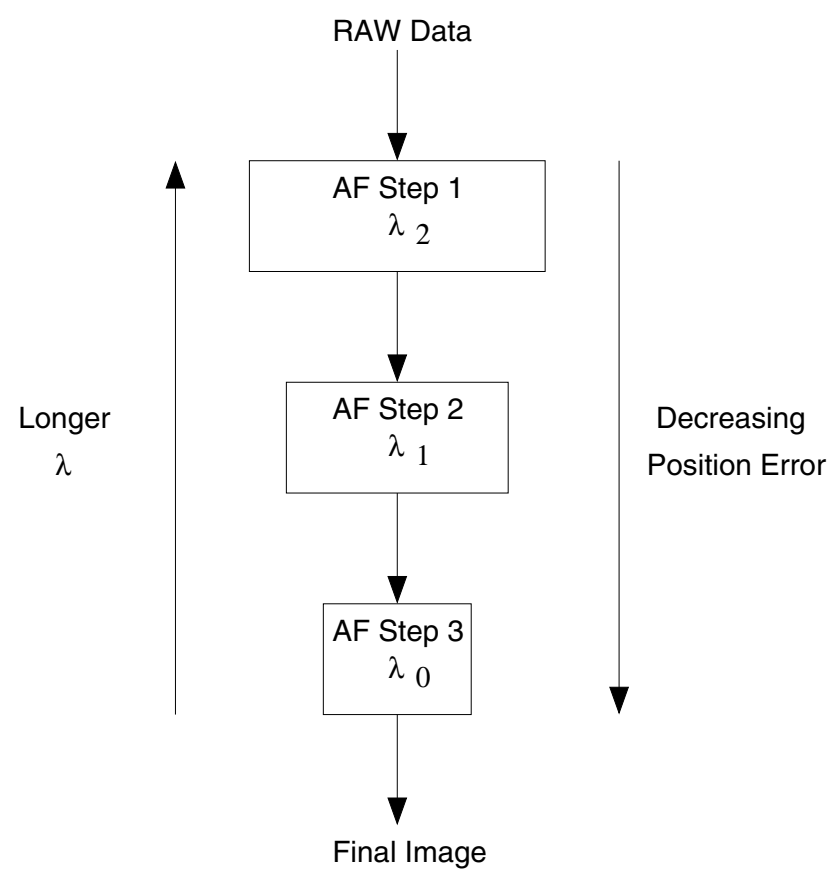

Figure 10: Auto-focus procedure with sub-band processing.

This step is repeated with decreasingly smaller wavelength and position error, until the original wavelength is used (Figure 10). The result is a faster progression through the solution surface, with lower probabilities of falling into local minima.

For image quality metric a quadratic entropy measure was used, which proved to be more robust and enable faster convergence than a first order entropy measure or a simple image contrast measure. For the entropy calculation, instead of assuming a specific probability density function, the probability density is estimated using the available data through a Panzer window method [11].

This auto-focus method is suitable for system working with an under-sampled swath and few transducers. No special image features are necessary for the algorithm to converge.

\section{INTERFEROMETRY WITH SUB-BAND PROCESSING}

One of the major issues with height mapping estimation using interferometry is the correct phase unwrapping under low signal to noise ratio, especially in cases where there are many wraps.

This problem is more complex with smaller wavelengths, since they will produce more wraps for the same height variation. 


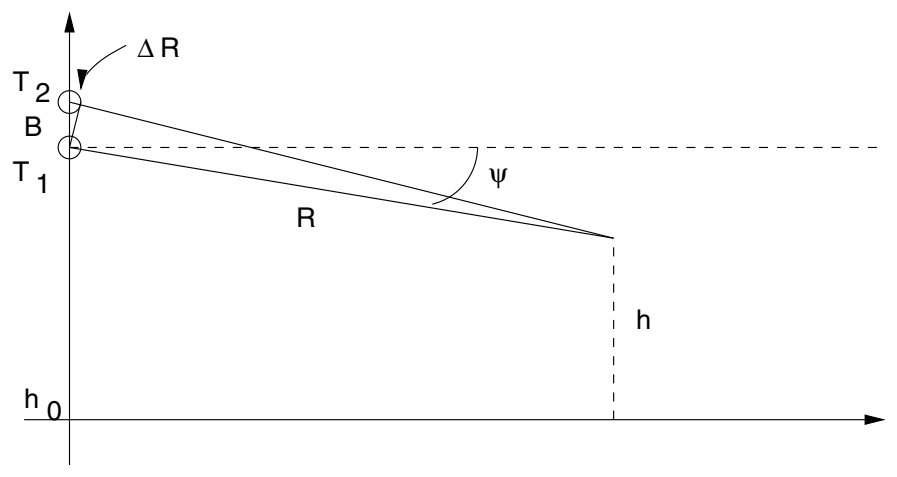

Figure 11: Schematic of the interferometric height mapping model.

Looking at Figure 11 and assuming that the range and distance between the sensors (B) is much larger than the difference in range due to height $(\Delta R)$, the height estimation is given by:

$$
h=h_{0}-R \sin \psi=h_{0}-R \frac{\Delta R}{B}
$$

And the range difference that enables the height computation is given by:

$$
\Delta R=\frac{(\phi+2 \pi n)}{2 \pi} \lambda
$$

Here $\phi$ is the measured phase difference and $\mathrm{n}$ the number of integer wraps. So we see that for a $\Delta \mathrm{R}$ resulting from the same height variation, the longer the wavelength, the smaller the phase variation and thus fewer wraps around $\pi$.
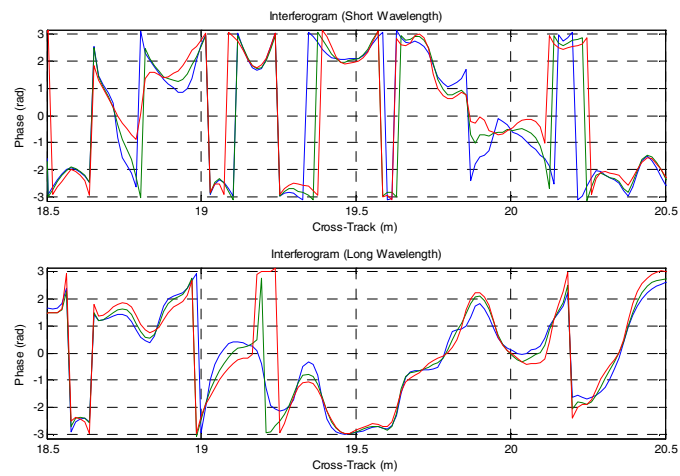

Figure 12: Two interferograms: one produced with a short wavelength signal and the other with a long wavelength signal.

Figure 12 is presents two interferograms. Both were produced using synthetic aperture images obtained in a test mission and derived from sub-band processing but with different wavelengths. It is evident here that with a longer wavelength the number of total wraps is smaller for the same extension and height variation, thus easing the subsequent unwrapping step.

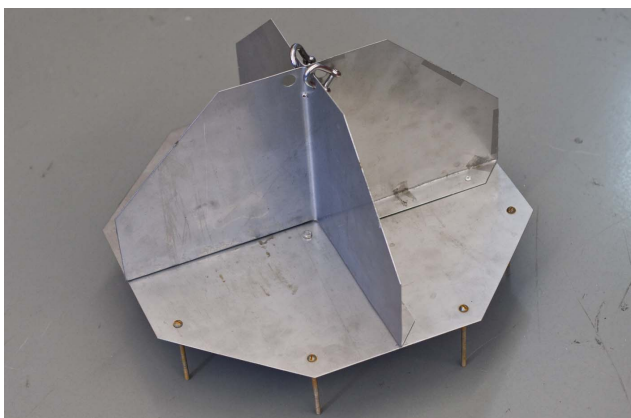

Figure 13: The artificial target used in the test missions.

\section{RESUlts}

To test the proposed method, several tests were carried in the Douro River, Portugal. An artificial target (Figure 13) was placed in the muddy river bottom which enabled to test the system aperture synthesis capabilities, resolution and autofocus. The artificial target is presented in Figure 13. It measures $20 \times 20 \times 20 \mathrm{~cm}$, but the target response seen by the sonar should be like a point after correct image synthesis. The sonar system is mounted on an autonomous boat that is capable of following predefine paths $([5,6,7])$ and maintain a specified target velocity. It moves at a nominal speed of $0.5 \mathrm{~m} / \mathrm{s}$, the transmitted signal center frequency is $200 \mathrm{kHz}$ and the chirp bandwidth is $30 \mathrm{kHz}$. It uses one transducer for signal transmission and two other in a vertical arrangement for reception. The transducers beam width is $18^{\circ}$ and the pulse repletion frequency used is $10 \mathrm{~Hz}$, which means that the swath is under-sampled in the along-track dimension by a factor of 4 . The system carries a real-time kinetic differential GPS system that together with an inertial unit provides and a digital compass provides a high precision navigation solution (maximum estimated error less than $1.5 \mathrm{~cm}$ ).
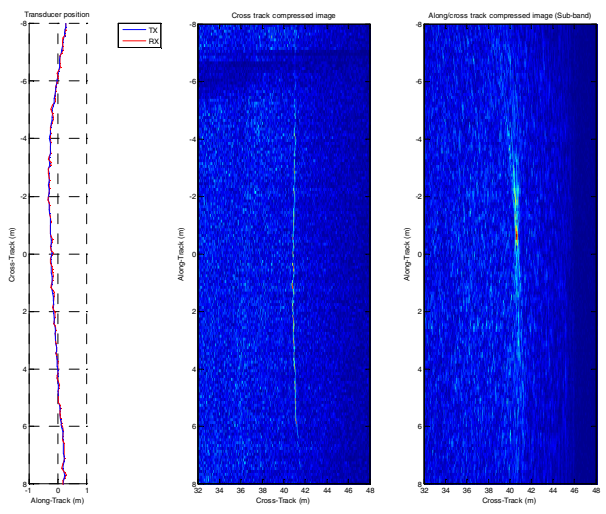

Figure 14: Sonar platform long-track positions, cross-track compressed image and resulting synthetic aperture image processed with sub-band technique. 
The pulse-compressed data is processed using this navigation data with a back-projection algorithm to form the synthetic aperture images.

In Figure 14 is presented one of the passages through the target. The sonar movement has quite a deviation from a linear motion which translates into the wavy hyperbole in the pulse compressed image. Using sub-band processing with a band division by 2 , it is possible to reconstruct the image forming a point.

This image can be, of course, enhanced through the described auto-focus procedures.

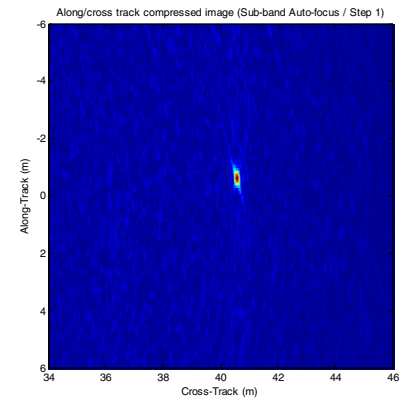

Figure 15: Artificial target: auto-focus step 1, wavelength $=40 \mathrm{~cm}$.

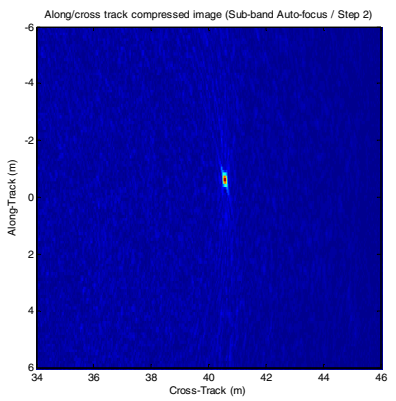

Figure 16: Artificial target: auto-focus step 2, wavelength $=20 \mathrm{~cm}$.

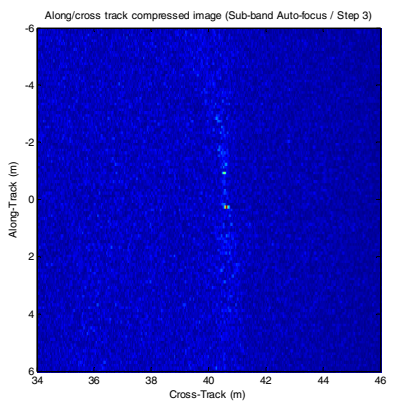

Figure 17: Artificial target: auto-focus step 3, final step, wavelength $=0.75 \mathrm{~cm}$.
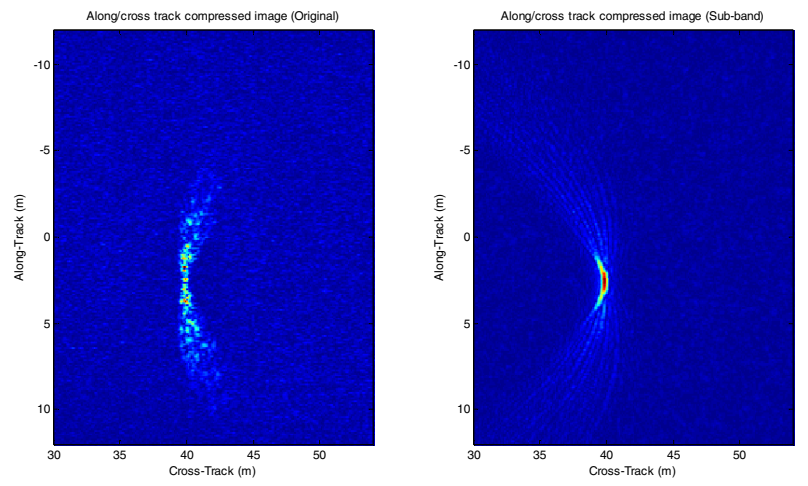

Figure 18: Another look at the artificial target in the river bed. With and without subband processing.

After the first auto-focus step, the enhancement is already quite visible (Figure 15), but it is necessary to proceed with a fine tuning of the auto-focus parameters with shorter wavelengths (Figure 16). At the final step (Figure 17), the data set uses the original wavelength and so it is possible to obtain the full resolution of the sonar system.

Figure 18 shows another passage through the target. Forming the synthetic image directly with the original wavelength $(0.75 \mathrm{~cm})$ is hardly feasible, the navigation motion errors obstruct the correct formation does giving origin to an unfocused image. With sub-band processing, however, it is possible to obtain an acceptable focused image, although with lower resolution.

Figure 19 shows an image obtained through this described process near a harbor entrance in the Douro River.

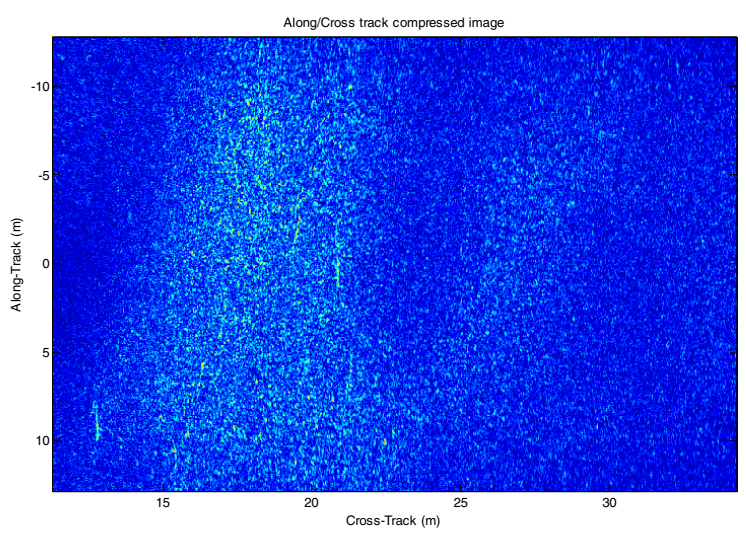

Figure 19: Reflectivity map of harbor entrance.

\section{CONCLUSION}

With the presented method it is possible to mitigate some of the problems that hinder high resolution synthetic aperture sonar. The use of high frequency signals imposes demanding restrictions in motion estimation and medium stability due to 
the sensibility of the image formation process to phase errors. A clever combination of the received signals enables the creation of a new one with equivalent frequency the equal to difference of the center frequencies of the previous ones. This longer wavelength signal effectively hides phase uncertainties and enables efficient auto-focus of the synthetic aperture sonar image. Moreover it is possible to apply this technique to interferometric height mapping, reducing the number of wraps in the interferogram.

\section{ACKNOWLEDGMENT}

This project has been possible thanks to the contributions of several institutions. The first author is supported by the FCT through the Ph.D. scholarship SFRH/BD/19976/2004/5R38. The authors would like to emphasize the support given by CIIMAR in financing part of the described system.

\section{REFERENCES}

[1] P. T. Gough, "Unified Framework for Modern Synthetic Aperture Imaging Algorithms". The International Journal of Imaging Systems and Technology, Vol. 8, pp. 343-358.

[2] Silkaitis, J.M.; Douglas, B.L.; Hua Lee, "Synthetic-aperture sonar imaging: system analysis, image formation, and motion compensation," Signals, Systems and Computers, 1995. 1995 Conference Record of the Twenty-Ninth Asilomar Conference on , vol.1, no., pp.423-427 vol.1, 30 Oct-1 Nov 1995.
[3] Fortune, S.A.; Hayes, M.P.; Gough, P.T., "Statistical autofocus of synthetic aperture sonar images using image contrast optimisation," OCEANS, 2001. MTS/IEEE Conference and Exhibition , vol.1, no., pp.163-169 vol.1, 2001.

[4] Shippey, G.; Banks, S.; Pihl, J., "SAS image reconstruction using Fast Polar Back Projection: comparisons with Fast Factored Back Projection and Fourier-domain imaging," Oceans 2005 - Europe , vol.1, no., pp. 96101 Vol. 1, 20-23 June 2005.

[5] N. Cruz, A. Matos, S. Cunha, S. Silva, "ZARCO - An Autonomous Craft for Underwater Surveys", Proceedings of the 7th Geomatic Week, Barcelona, Spain, Feb 2007.

[6] S. Silva, S. Cunha, A. Matos, N. Cruz, "An In-SAS System For Shallow Water Surveying", Proceedings of the 7th Geomatic Week, Barcelona, Spain, Feb 2007.

[7] Silva, S.R.; Cunha, S.; Matos, A.; Cruz, N., "An Autonomous Boat Based Synthetic Aperture Sonar," Oceans 2007, vol., no., pp.1-7, Sept. 29 2007Oct. 42007.

[8] McHugh, R.; Shaw, S.; Taylor, N., "Spatial sampling ambiguities in synthetic aperture sonar for broadside and squint look operations," OCEANS '98 Conference Proceedings, vol.2, no., pp.960-964 vol.2, 28 Sep-1 Oct 1998.

[9] Tomiyasu, K., "Tutorial review of synthetic-aperture radar (SAR) with applications to imaging of the ocean surface," Proceedings of the IEEE, vol.66, no.5, pp. 563-583, May 1978.

[10] Fornaro, G., "Trajectory deviations in airborne SAR: analysis and compensation ," Aerospace and Electronic Systems, IEEE Transactions on, vol.35, no.3, pp.997-1009, Jul 1999.

[11] W. Liu, P. P. Pokharel, J. C. Principe, "Correntropy: A Localized Similarity Measure", International Joint Conference on Neural Networks, 2006, pp. $4919-4924$.

[12] Cutrona, Louis J., "Comparison of sonar system performance achievable using synthetic-aperture techniques with the performance achievable by more conventional means", The Journal of the Acoustical Society of America, Volume 58, Issue 2, August 1975, pp.336-348. 